\title{
Qualidade da assistência à saúde bucal na atenção primária no Brasil
}

\author{
I 1 Marília Guedes da Silveira Arrais, ${ }^{2}$ Angelo Giuseppe Roncalli, \\ ${ }^{3}$ Tatyana Souza Rosendo I
}

Resumo: O objetivo desta pesquisa foi analisar a qualidade da assistência à saúde bucal na Atenção Primária em Saúde no Brasil e identificar fatores contextuais socioeconômicos associados. Trata-se de um estudo seccional que teve como unidade de análise todas as equipes de saúde bucal que participaram da avaliaçáo externa do segundo ciclo do Programa de Melhoria do Acesso e Qualidade - Atenção Básica. A qualidade foi medida nas dimensôes: acesso, resolutividade, processo de trabalho, coordenação do cuidado e infraestrutura. Os critérios de qualidade da avaliação externa foram agrupados nas dimensôes citadas e ponderados pela técnica de Teoria de Resposta ao Item. O indicador de qualidade da Saúde Bucal foi resultado do agrupamento das variáveis latentes. Foram realizadas análises descritivas por regióes e de associação entre "Qualidade da Assistência em Saúde Bucal" e o Índice de Desenvolvimento Humano Municipal, com significância de $5 \%$. Apenas 25\% das equipes de saúde bucal são consideradas boas. A dimensão infraestrutura foi melhor avaliada e a coordenação do cuidado, pior. Não há diferença estatisticamente significativa na análise entre os municípios com alto e baixo IDH. Analisar a qualidade é primordial para que se realize a melhora contínua da qualidade dos cuidados em saúde.

> Palavras-chave: Saúde Bucal; Avaliação da qualidade dos cuidados de saúde; Atenção Primária à Saúde.

\author{
${ }^{1}$ Mestrado Profissional em Saúde \\ da Família, RENASF. Universidade \\ Federal do Rio Grande do Norte. \\ Natal-RN, Brasil (marilia.arrais@ \\ ifce.edu.br). \\ ORCID: 0000-0002-9273-6845 \\ ${ }^{2}$ Programa de Pós-graduação \\ em Saúde Coletiva. Universidade \\ Federal do Rio Grande do Norte. \\ Natal-RN, Brasil (roncalli@terra. \\ com.br). \\ ORCID: 0000-0001-5311-697X \\ ${ }^{3}$ Departamento de Saúde \\ Coletiva. Universidade Federal do \\ Rio Grande do Norte. Natal-RN, \\ Brasil (tatyana.rosendo@ufrn.br). \\ ORCID: 0000-0001-6131-3201
}




\section{Introdução}

Apesar das melhorias advindas do processo de ampliação e reorganização da atenção primária em saúde bucal no Brasil, esse cenário ainda não foi capaz de reverter os problemas no acesso e utilização dos serviços, por muitas vezes persistindo o caráter curativo e mutilador da assistência odontológica (AQUILANTE e ACIOLE, 2015). Bulgareli et al. (2014) afirmam que a atenção à saúde bucal no Brasil tem-se caracterizado pela insuficiência de procedimentos coletivos e preventivos individuais e pela baixa cobertura de procedimentos curativos e de urgência (BULGARELI et al., 2014). Neste sentido, há um movimento internacional no qual a melhoria da qualidade dos serviços tem sido destacada como fundamental. Assim sendo, os cuidados de saúde de alta qualidade sáo os cuidados certos, de forma coordenada, que respondam às necessidades e preferências dos usuários do serviço, minimizando danos e desperdício de recursos (OMS, 2018).

Em 2004, o Ministério da Saúde lançou a Política Nacional de Saúde Bucal Brasil Sorridente. Com essa política, a saúde bucal passou a ser ofertada de forma integral. Houve a inserção de procedimentos mais complexos na atenção primária e a criação de uma rede de serviços de atenção em saúde bucal no Sistema Único de Saúde (SUS) (BRASIL, 2011a). Outro aspecto importante é que a expansão da rede básica dos serviços de saúde bucal deve vir acompanhada de inovaçôes no modelo assistencial e no cotidiano do processo de trabalho das equipes, com a superação das práticas odontológicas centradas em procedimentos curativos e individuais (CORRÊA e CELESTE, 2015). Dentro desta ótica, Starfield (2002) sustenta que a atenção primária é a principal porta de entrada, coordenadora do cuidado e ordenadora das açóes e serviços disponibilizados na rede.

O último levantamento epidemiológico em saúde bucal de âmbito nacional, conhecido como SB-Brasil 2010, mostrou uma distribuição heterogênea da doença cárie, registrando diferenças entre as regiōes e os grupos sociais. Comparativamente ao observado em 2003, este padrão de diferenças regionais se manteve (BRASIL, 2011). Já para Scherer e Scherer (2015) as diferenças regionais na prevalência e gravidade da cárie são marcantes, o que indica necessidade de políticas voltadas para a equidade na atençáo. Apesar dos avanços, a erradicação da pobreza e a redução das desigualdades continuam a ser parte dos principais desafios para o desenvolvimento humano e sustentável. No país, embora tenha ocorrido a redução 
das desigualdades entre os diferentes grupos sociais, ainda é bastante evidente a existência das disparidades regionais (IPEA, 2019).

No contexto da avaliação dos serviços, um documento publicado recentemente pela Organização Mundial de Saúde sobre a qualidade dos serviços aponta que cuidados de má qualidade não são apenas prejudiciais, mas também desperdiçam recursos que podem ser investidos em outras importantes áreas do desenvolvimento social e econômico para a vida dos cidadãos (OMS, 2018). Já a definição de alta qualidade, proposta pela Comissão de Saúde Global, é que um sistema de saúde de alta qualidade é aquele que otimiza a saúde e o cuidado em um determinado contexto, que melhora ou mantém os resultados de saúde, sendo valorizado e confiado por todas as pessoas, além de responder às necessidades populacionais (KRUK et al., 2018). Assim sendo, a qualidade da atenção à saúde bucal é entendida como a melhora permanente da qualidade tanto dos serviços, quanto do processo de trabalho das açóes ofertadas aos usuários.

No Brasil, para estimular a ampliação do acesso e a melhoria da qualidade da atenção básica, no ano de 2011, foi lançado o Programa de Melhoria do Acesso e da Qualidade na Atenção Básica (PMAQ) - AB, que trata-se de um modelo de avaliação de desempenho dos sistemas de saúde, o qual pretende mensurar os possíveis efeitos da política de saúde com vistas a subsidiar a tomada de decisão, garantir a transparência dos processos de gestão do SUS e dar visibilidade aos resultados alcançados, além de fortalecer o controle social e o foco do sistema de saúde nos usuários. Com relação à adesão, todas as equipes de saúde da atenção básica, incluindo as equipes de saúde bucal, em diferentes modalidades, podem aderir ao PMAQ (BRASIL, 2015).

Para analisar a qualidade da assistência à saúde bucal na atenção primária, identificamos os critérios que subsidiaram esta pesquisa a partir do relatório analítico do Banco de dados do PMAQ (Programa de Melhoria do Acesso e da Qualidade na Atenção Básica). O relatório supracitado é uma oferta do Ministério da Saúde aos trabalhadores e gestores e apresenta temas relevantes para a atenção básica como: acesso, resolutividade, coordenação do cuidado, processo de trabalho e gestão e apoio. Estes critérios contribuíram para a discussão e reflexão sobre a qualidade da assistência à saúde bucal segundo a análise deste estudo (BRASIL, 2019b).

Diante dessa realidade e inspirando-se no crescimento da institucionalização da avaliação e do monitoramento na atenção básica, optou-se por trabalhar com 
o tema "Qualidade da assistência à saúde bucal na atenção primária no Brasil", a partir do banco de dados do PMAQ-AB, porque através dele é possível analisar a qualidade da assistência à saúde bucal no Brasil e identificar se há associação com o nível de desenvolvimento econômico. Logo, a qualidade será analisada a partir das condiçôes de acesso, resolutividade, coordenação do cuidado, processo de trabalho e infraestrutura. E ainda, se há associação entre a qualidade da assistência à saúde bucal e o Índice de Desenvolvimento Humano (IDH-M) nos municípios brasileiros.

\section{Procedimentos Metodológicos}

\section{Tipo de pesquisa}

Para análise da qualidade da assistência à saúde bucal a partir da avaliação externa do PMAQ, decidiu-se pela realização de um estudo seccional, com abordagem quantitativa, no qual serão utilizados dados do segundo ciclo disponibilizados pelo Ministério da Saúde, a partir da dimensão voltada para o cuidado à saúde bucal em dois módulos do instrumento de coleta, sendo: módulo V - Observação na unidade e módulo VI - Entrevista com Profissional da Equipe de Atenção Básica. A avaliação externa do PMAQ-AB faz parte de uma pesquisa multicêntrica, realizada em todos os municípios brasileiros pelo MS em parceria com seis instituiçôes de pesquisa, que coletaram seus dados nos anos de 2012/2013, para o primeiro ciclo, 2013/2014 para o segundo ciclo e 2017/2018 para o terceiro ciclo.

\section{Universo e amostra}

O universo desta pesquisa será constituído por todas as Unidades de Saúde Bucal (Módulo V) e Equipes de Saúde Bucal (Módulo VI) que participaram da avaliação externa do segundo ciclo do PMAQ-AB.

\section{Procedimentos de coleta de dados}

Foram utilizados os dados secundários contidos nos bancos de avaliação externa do PMAQ-AB segundo ciclo, de livre acesso, disponibilizados pelo Ministério da Saúde, no site com o endereço eletrônico: http://dab.saude.gov.br/portaldab/ ape_pmaq.php?conteudo=2_ciclo. Ao ponto que foram coletados dados relativos à saúde bucal, presentes nos módulo V - Observação na unidade - e módulo VI Entrevista com Profissional da Equipe de Saúde Bucal e Verificação de Documentos na Unidade de Saúde. 


\section{Indicadores de qualidade}

Para a avaliação da qualidade dos serviços de saúde foram utilizados os seguintes indicadores de qualidade: Acesso, Resolutividade, Coordenação do Cuidado, Processo de Trabalho e Infraestrutura. Além disso, foi construído um indicador síntese que agrupou todas as dimensóes citadas em uma única medida, sendo a variável "Qualidade da assistência à saúde bucal". Esses indicadores foram construídos a partir da observação nas Unidades e entrevistas aos profissionais das Equipes (Módulos V e VI).

Para cada dimensão foram selecionados critérios da avaliação externa que tivessem relação com a assistência à saúde bucal, de acordo com o referencial teórico sobre o tema. Os critérios selecionados para compor cada dimensão estão descritos no Quadro 1.

Quadro 1. Critérios dos Módulos V (Observação na Unidade Básica de Saúde) e Módulo VI (Entrevista com Profissional da Equipe de Atenção Básica e Verificação de Documentos na Unidade de Saúde) por dimensóes.

\begin{tabular}{|l|l|l|}
\hline Dimensão & CÓDIGO & \multicolumn{1}{c|}{ Variáveis } \\
\hline \multirow{7}{*}{ Acesso } & VI.13.1 & $\begin{array}{l}\text { A agenda de atendimento odontológico garante a consulta por demanda e } \\
\text { agendamento? }\end{array}$ \\
\cline { 2 - 4 } & VI.13.6 & As consultas na Unidade são agendadas em qualquer dia e qualquer horário? \\
\cline { 2 - 4 } & VI.13.7 & $\begin{array}{l}\text { A Equipe de Saúde Bucal garante agendamento de retorno para a } \\
\text { continuidade do tratamento de um usuário que iniciou seu tratamento? }\end{array}$ \\
\cline { 2 - 4 } & VI.18.1 & $\begin{array}{l}\text { A Equipe de Saúde Bucal realiza acompanhamento das gestantes por meio de } \\
\text { consultas? }\end{array}$ \\
\cline { 2 - 4 } & VI.19.1 & $\begin{array}{l}\text { A Equipe de Saúde Bucal realiza atendimento em crianças de até 5 anos de } \\
\text { idade? }\end{array}$ \\
\hline
\end{tabular}

continua... 


\begin{tabular}{|c|c|c|}
\hline Dimensão & CÓDIGO & Variáveis \\
\hline \multirow{19}{*}{ Resolutividade } & VI.16.5.1 & A Equipe de Saúde Bucal realiza drenagem de abscesso? \\
\hline & VI.16.5.2 & A Equipe de Saúde Bucal realiza sutura de ferimentos por trauma? \\
\hline & VI.16.5.3 & A ESB realiza remoção de dentes impactados? \\
\hline & VI.16.5.4 & A ESB realiza frenectomia? \\
\hline & VI.16.5.5 & A ESB realiza remoçâo de cistos? \\
\hline & VI.16.5.6 & A ESB realiza acesso a polpa dentária \\
\hline & VI.16.5.7 & A ESB realiza aplicação tópica de flúor? \\
\hline & VI.16.5.8 & A ESB realiza exodontia de dente decíduo? \\
\hline & VI.16.5.9 & A ESB realiza exodontia de dente permanente? \\
\hline & VI.16.5.10 & A ESB realiza restauração de amálgama? \\
\hline & VI.16.5.11 & A ESB realiza restauração de resina composta? \\
\hline & VI.16.5.12 & A ESB realiza restauração de dente decíduo? \\
\hline & VI.16.5.13 & A ESB realiza pulpotomia? \\
\hline & VI.16.5.14 & A ESB realiza raspagem, alisamento e polimento supra gengivais? \\
\hline & VI.16.5.15 & A ESB realiza tratamento da alveolite? \\
\hline & VI.16.5.16 & A ESB realiza ulotomia/ulectomia? \\
\hline & VI.16.5.17 & A ESB realiza cimentação de prótese? \\
\hline & VI.16.6 & A ESB realiza tratamento concluído? \\
\hline & VI.17.3 & $\begin{array}{l}\text { A Equipe de Saúde Bucal realiza açôes de prevenção e detecção do câncer de } \\
\text { boca? }\end{array}$ \\
\hline \multirow{8}{*}{$\begin{array}{l}\text { Processo de } \\
\text { Trabalho }\end{array}$} & VI.7.1 & $\begin{array}{l}\text { A equipe realiza atividade de planejamento e programação de suas atividades } \\
\text { mensalmente? }\end{array}$ \\
\hline & VI.7.2 & $\begin{array}{l}\text { A Equipe de Saúde Bucal realiza monitoramento e análise dos indicadores e } \\
\text { informaçóes de saúde bucal? }\end{array}$ \\
\hline & VI.7.3 & $\begin{array}{l}\text { A ESB recebe apoio para o planejamento e organização do processo de } \\
\text { trabalho? }\end{array}$ \\
\hline & VI.7.4 & $\begin{array}{l}\text { A gestão disponibiliza para a ESB informaçôes que auxiliem a análise de } \\
\text { situação de saúde? }\end{array}$ \\
\hline & VI.7.5 & $\begin{array}{l}\text { Foi realizado algum processo de autoavaliação pela Equipe de Saúde Bucal } \\
\text { nos últimos seis meses? }\end{array}$ \\
\hline & VI.13.5 & $\begin{array}{l}\text { A agenda da Equipe de Saúde Bucal está organizada para ofertar atividades de } \\
\text { educação em saúde bucal no território? }\end{array}$ \\
\hline & VI.20.1 & A Equipe de Saúde Bucal realiza visita domiciliar? \\
\hline & VI.22.1 & A Equipe de Saúde Bucal realiza atividades na escola/creche? \\
\hline
\end{tabular}




\begin{tabular}{|c|c|c|}
\hline Dimensão & CÓDIGO & Variáveis \\
\hline \multirow{6}{*}{$\begin{array}{l}\text { Coordenação } \\
\text { do Cuidado }\end{array}$} & VI.14.1 & $\begin{array}{l}\text { A equipe de saúde bucal tem disponível, por parte da rede de saúde, oferta de } \\
\text { consultas especializadas? }\end{array}$ \\
\hline & VI.14.4 & $\begin{array}{l}\text { A Equipe de Saúde Bucal mantém registrado os usuários encaminhados para } \\
\text { outros pontos de atenção? }\end{array}$ \\
\hline & VI.15.1 & $\begin{array}{l}\text { Existe central de regulação disponível para o encaminhamento dos usuários } \\
\text { para as especialidades odontológicas? }\end{array}$ \\
\hline & VI.15.2 & $\begin{array}{l}\text { Com que frequência os profissionais da Equipe de Saúde Bucal entram em } \\
\text { contato com especialistas para trocar informaçôes sobre os seus pacientes } \\
\text { encaminhados? }\end{array}$ \\
\hline & VI.15.3 & Existe algum fluxo de comunicação institucionalizado? \\
\hline & VI.17.2 & $\begin{array}{l}\text { A Equipe de Saúde Bucal possui referência para encaminhar os casos } \\
\text { suspeitos/confirmados de câncer de boca? }\end{array}$ \\
\hline \multirow{17}{*}{ Infraestrutura } & V 7.1 & Possui amalgamador \\
\hline & V 7.2 & Possui aparelho de RX odontológico \\
\hline & V 7.4 & Possui autoclave \\
\hline & V 7.5 & Possui avental de chumbo \\
\hline & V 7.6 & Possui cadeira odontológica \\
\hline & V 7.7 & Possui cuspideira \\
\hline & V 7.8 & Possui sugador \\
\hline & V 7.9 & Possui refletor \\
\hline & V 7.10 & Possui mocho \\
\hline & V 7.12 & Possui caneta de alta rotação \\
\hline & V 7.13 & Possui caneta de baixa rotação \\
\hline & V 7.14 & Possui compressor de ar com válvula de segurança \\
\hline & V 7.15 & Possui fotopolimerizador \\
\hline & V 7.19 & Possui caixa de revelação \\
\hline & V 9.9 & Possui brocas diversas \\
\hline & V 9.12 & Possui luvas, óculos, mascaras, aventais, tocas \\
\hline & V 9.15 & Possui fixador e revelador para radiografia \\
\hline
\end{tabular}

Fonte: Brasil, 2018.

Realizou-se um linkage dos bancos do módulo V com o módulo VI a partir da variável do Cadastro Nacional dos Estabelecimentos de Saúde (CNES). Como o banco do módulo $\mathrm{V}$ possuía menor quantidade de dados, porque contém apenas 
informações por unidade de saúde, ao fazer o linkage, as informaçôes das unidades se repetiram para as equipes quando havia mais de uma equipe por unidade de saúde.

Os critérios foram ponderados a posteriori pela Teoria de Resposta ao Item (TRI), que avaliou o poder discriminatório de cada um deles. Critérios com baixo poder discriminatório foram excluídos, de modo que ao final, foram mantidos critérios com capacidade de discriminar as diferenças existentes entre as equipes analisadas. Além disso, foram excluídos critérios que possuíram poder discriminatório muito alto que impediu o ajuste do modelo.

Foi obtida, para cada dimensão, uma variável latente ponderada pelo poder discriminatório dos critérios, sintetizando todos eles em uma única medida. Em seguida, foram formados clusters dessa variável latente, que foram classificados em três categorias: 1 = Boa Qualidade, 2 =Moderada Qualidade e 3=Baixa Qualidade. O indicador síntese de Qualidade da Assistência à Saúde da Bucal foi obtido a partir do agrupamento das variáveis latentes das quatro dimensôes que também foram agrupados nas três categorias citadas acima.

Ao final, realizou-se novamente mais um linkage no banco, desta vez com o banco de variáveis contextuais do Índice de Desenvolvimento Humano Municipal (IDH-M) (PNUD, 2013).

Para a análise de associação com os indicadores da qualidade da assistência, a variável contextual das características socioeconômicas utilizada foi o Índice de Desenvolvimento Humano Municipal (IDH-M). Esta variável foi obtida a partir dos bancos de dados do Programa das Naçóes Unidas para o Desenvolvimento (PNUD). (PNUD,2013).

\section{Análise dos dados}

A análise descritiva foi realizada através de frequências absolutas e relativas, na qual foi observada a qualidade por dimensão e a qualidade da assistência à saúde bucal no geral, segundo as regiôes do Brasil. A análise de associação escolhida foi a regressão de Poisson multinível, com interceptos variáveis, tendo como variáveis dependentes a "Qualidade da Assistência em Saúde Bucal”. A variável independente do nível contextual (municípios) foi o IDH-M. Para a análise bivariada as variáveis dependentes e independentes foram dicotomizadas. Todas as análises de associação consideraram a significância de 5\%. 


\section{Consideraçôes éticas}

A pesquisa foi submetida ao Comitê de Ética e Pesquisa do Hospital Universitário Onofre Lopes da Universidade Federal do Rio Grande do Norte (HUOL/UFRN) e foi aprovada com parecer $\mathrm{n}^{\mathrm{o}}: 2.932 .991 / 18$.

\section{Resultados}

Neste estudo, 18.114 equipes de saúde bucal no Brasil foram avaliadas. No tocante a tabela 1 , seguem os critérios dos módulos V e VI por dimensōes e respectivos coeficientes e intervalos de confiança. No que concerne à dimensão acesso, foram selecionadas 05 variáveis, porém 03 delas foram excluídas devido ao seu baixo poder discriminatório. No entanto, de acordo com os resultados construídos neste estudo, as varáveis VI.18.1 e VI.19.1 não geraram a variável latente por não conseguirem ajuste no modelo estatístico na TRI. Portanto, esta dimensão foi excluída da análise.

Ao tratarmos sobre a dimensão resolutividade foram selecionados 19 critérios, porém 8 destes foram excluídos por conta de seu baixo poder discriminatório. Quanto à dimensão processo de trabalho, foram selecionados 08 critérios, porém 02 destes foram excluídos pelo mesmo motivo. Para os achados sobre a dimensão coordenação do cuidado foram selecionadas 06 variáveis, no entanto, um critério foi excluído devido ao seu baixo poder para realizar discriminaçáo. Por fim, para o estudo da dimensão infraestrutura foram selecionados 17 critérios, porém 6 destes também foram excluídos em razão do seu baixo poder discriminatório. Os critérios que possuem baixo poder discriminatório foram os que apresentaram resultados menor que um. 
Tabela 1. Variáveis dos Módulos V (Observação na Unidade Básica de Saúde) e Módulo VI (Entrevista com Profissional da Equipe de Atenção Básica e Verificação de Documentos na Unidade de Saúde) por dimensôes.

\begin{tabular}{|c|c|c|c|c|}
\hline Dimensão & CÓDIGO & Variáveis & Coeficiente & $\begin{array}{l}\text { Intervalo de } \\
\text { confiança }\end{array}$ \\
\hline \multirow{5}{*}{ Acesso* } & VI.13.1 & $\begin{array}{l}\text { A agenda de atendimento odontológico } \\
\text { garante a consulta por demanda e } \\
\text { agendamento? }\end{array}$ & -- & -- \\
\hline & VI.13.6 & $\begin{array}{l}\text { As consultas na Unidade são agendadas } \\
\text { em qualquer dia e qualquer horário? }\end{array}$ & -- & -- \\
\hline & VI.13.7 & $\begin{array}{l}\text { A Equipe de Saúde Bucal garante } \\
\text { agendamento de retorno para a } \\
\text { continuidade do tratamento de um } \\
\text { usuário que iniciou seu tratamento? }\end{array}$ & -- & -- \\
\hline & VI.18.1 & $\begin{array}{l}\text { A Equipe de Saúde Bucal realiza } \\
\text { acompanhamento das gestantes por meio } \\
\text { de consultas? }\end{array}$ & -- & -- \\
\hline & VI.19.1 & $\begin{array}{l}\text { A Equipe de Saúde Bucal realiza } \\
\text { atendimento em crianças de até } 5 \text { anos de } \\
\text { idade? }\end{array}$ & -- & -- \\
\hline \multirow{11}{*}{ Resolutividade } & VI.16.5.1 & $\begin{array}{l}\text { A Equipe de Saúde Bucal realiza } \\
\text { drenagem de abscesso? }\end{array}$ & 1.16 & $1.08-1.24$ \\
\hline & VI.16.5.6 & A ESB realiza acesso a polpa dentária & 2.23 & $2.08-2.38$ \\
\hline & VI.16.5.7 & A ESB realiza aplicação tópica de flúor? & 1.54 & $1.38-1.69$ \\
\hline & VI.16.5.8 & A ESB realiza exodontia de dente decíduo? & 2.54 & $2.29-2.78$ \\
\hline & VI.16.5.9 & $\begin{array}{l}\text { A ESB realiza exodontia de dente } \\
\text { permanente? }\end{array}$ & 2.04 & $1.86-2.22$ \\
\hline & VI.16.5.11 & $\begin{array}{l}\text { A ESB realiza restauraçấo de resina } \\
\text { composta? }\end{array}$ & 1.72 & $1.57-1.87$ \\
\hline & VI.16.5.12 & $\begin{array}{l}\text { A ESB realiza restauração de dente } \\
\text { decíduo? }\end{array}$ & 2.75 & $2.47-3.02$ \\
\hline & VI.16.5.13 & A ESB realiza pulpotomia? & 2.09 & $1.95-2.23$ \\
\hline & VI.16.5.14 & $\begin{array}{l}\text { A ESB realiza raspagem, alisamento e } \\
\text { polimento supra gengivais? }\end{array}$ & 1.80 & $1.65-1.94$ \\
\hline & VI.16.5.15 & A ESB realiza tratamento da alveolite? & 1.66 & $1.55-1.77$ \\
\hline & VI.16.5.16 & A ESB realiza ulotomia/ulectomia? & 1.17 & $1.10-1.24$ \\
\hline
\end{tabular}

continua... 


\begin{tabular}{|c|c|c|c|c|}
\hline Dimensão & CÓDIGO & Variáveis & Coeficiente & $\begin{array}{l}\text { Intervalo de } \\
\text { confiança }\end{array}$ \\
\hline \multirow{6}{*}{$\begin{array}{l}\text { Processo de } \\
\text { Trabalho }\end{array}$} & VI.7.1 & $\begin{array}{l}\text { A equipe realiza atividade de } \\
\text { planejamento e programação de suas } \\
\text { atividades mensalmente? }\end{array}$ & 1.19 & $1.12-1.26$ \\
\hline & VI.7.2 & $\begin{array}{l}\text { A Equipe de Saúde Bucal realiza } \\
\text { monitoramento e análise dos indicadores e } \\
\text { informaçōes de saúde bucal? }\end{array}$ & 1.88 & $1.79-1.97$ \\
\hline & VI.7.3 & $\begin{array}{l}\text { A ESB recebe apoio para o planejamento e } \\
\text { organizaçáo do processo de trabalho? }\end{array}$ & 2.79 & $2.63-2.95$ \\
\hline & VI.7.4 & $\begin{array}{l}\text { A gestão disponibiliza para a ESB } \\
\text { informaçóes que auxiliem a análise de } \\
\text { situação de saúde? }\end{array}$ & 3.24 & $3.02-3.47$ \\
\hline & VI.13.5 & $\begin{array}{l}\text { A agenda da Equipe de Saúde Bucal está } \\
\text { organizada para ofertar atividades de } \\
\text { educaçáo em saúde bucal no território? }\end{array}$ & 1.43 & $1.34-1.52$ \\
\hline & VI.22.1 & $\begin{array}{l}\text { A Equipe de Saúde Bucal realiza } \\
\text { atividades na escola/creche? }\end{array}$ & 1.28 & $1.19-1.36$ \\
\hline \multirow{5}{*}{$\begin{array}{l}\text { Coordenação } \\
\text { do Cuidado }\end{array}$} & VI.14.1 & $\begin{array}{l}\text { A equipe de saúde bucal tem disponível, } \\
\text { por parte da rede de saúde, oferta de } \\
\text { consultas especializadas? }\end{array}$ & 2.55 & $2.40-2.69$ \\
\hline & VI.15.1 & $\begin{array}{l}\text { Existe central de regulação disponível } \\
\text { para o encaminhamento dos usuários para } \\
\text { as especialidades odontológicas? }\end{array}$ & 1.14 & $1.09-1.20$ \\
\hline & VI.15.2 & $\begin{array}{l}\text { Com que frequência os profissionais } \\
\text { da Equipe de Saúde Bucal entram em } \\
\text { contato com especialistas para trocar } \\
\text { informaçóes sobre os seus pacientes } \\
\text { encaminhados? }\end{array}$ & 2.16 & $2.05-2.27$ \\
\hline & VI.15.3 & $\begin{array}{l}\text { Existe algum fluxo de comunicação } \\
\text { institucionalizado? }\end{array}$ & 2.32 & $2.19-2.45$ \\
\hline & VI.17.2 & $\begin{array}{l}\text { A Equipe de Saúde Bucal possui referência } \\
\text { para encaminhar os casos suspeitos/ } \\
\text { confirmados de câncer de boca? }\end{array}$ & 1.32 & $1.25-1.39$ \\
\hline
\end{tabular}

continua... 


\begin{tabular}{|c|c|c|c|c|}
\hline Dimensáo & CÓDIGO & Variáveis & Coeficiente & $\begin{array}{l}\text { Intervalo de } \\
\text { confiança }\end{array}$ \\
\hline \multirow{11}{*}{ Infraestrutura } & V 7.6 & Possui cadeira odontológica? & 3.71 & $3.21-4.21$ \\
\hline & V 7.7 & Possui cuspideira? & 1.85 & $1.67-2.02$ \\
\hline & V 7.8 & Possui sugador? & 2.94 & $2.62-3.26$ \\
\hline & V 7.9 & Possui refletor? & 3.89 & $3.34-4.44$ \\
\hline & V 7.10 & Possui mocho? & 2.55 & $2.28-2.81$ \\
\hline & V 7.12 & Possui caneta de alta rotaçáo? & 3.31 & $2.93-3.69$ \\
\hline & V 7.13 & Possui caneta de baixa rotaçáo? & 1.62 & $1.47-1.76$ \\
\hline & V 7.14 & $\begin{array}{l}\text { Possui compressor de ar com válvula de } \\
\text { segurança? }\end{array}$ & 1.96 & $1.78-2.14$ \\
\hline & V 7.15 & Possui fotopolimerizador? & 1.98 & $1.80-2.16$ \\
\hline & V 9.9 & Possui brocas diversas? & 1.91 & $1.73-2.10$ \\
\hline & V 9.12 & $\begin{array}{l}\text { Possui luvas, óculos, máscaras, aventais, } \\
\text { tocas? }\end{array}$ & 1.67 & $1.51-1.83$ \\
\hline
\end{tabular}

* A dimensão Acesso e acolhimento não teve seus coeficientes de discriminação, nem seus respectivos Intervalos de Confiança, gerados pela TRI devido ao não ajuste do modelo.

Fonte: Próprio autor.

A Tabela 2 apresenta a distribuição das equipes de saúde segundo a qualidade da assistência à saúde bucal por dimensões analisadas e por regióes brasileiras.

Tabela 2. Distribuição das equipes de saúde segundo a qualidade da assistência à saúde bucal por dimensóes analisadas e por regiôes brasileiras. Natal, RN, 2019.

\begin{tabular}{lcccccc}
\hline DIMENSÃO & BRASIL & NORTE & NORDESTE & $\begin{array}{c}\text { CENTRO } \\
\text { OESTE }\end{array}$ & SUDESTE & SUL \\
\hline Saúde Bucal & $\mathrm{N}(\%)$ & $\mathrm{N}(\%)$ & $\mathrm{N}(\%)$ & $\mathrm{N}(\%)$ & $\mathrm{N}(\%)$ & $\mathrm{N}(\%)$ \\
\hline Boa & $4448(25,1 \%)$ & $409(32,9 \%)$ & $1624(21,5 \%)$ & $515(33,4 \%)$ & $1182(24,2 \%)$ & $718(29,1 \%)$ \\
\hline Regular & $9414(53,2 \%)$ & $373(30,0 \%)$ & $3649(48,3 \%)$ & $643(41,7 \%)$ & $3206(65,5 \%)$ & $1543(62,5 \%)$ \\
\hline Ruim & $3845(21,7 \%)$ & $461(37,1 \%)$ & $2289(30,3 \%)$ & $385(25,0 \%)$ & $503(10,3 \%)$ & $207(8,4 \%)$ \\
\hline TOTAL & $17707(100 \%)^{*}$ & $1243(100 \%)^{*}$ & $7562(100 \%)^{*}$ & $1543(100 \%)^{*}$ & $4891(100 \%)^{*}$ & $2468(100 \%)^{*}$ \\
\hline
\end{tabular}




\begin{tabular}{|c|c|c|c|c|c|c|}
\hline \multirow[t]{2}{*}{ DIMENSÃO } & \multicolumn{6}{|c|}{ BRASIL/ REGIÃO } \\
\hline & BRASIL & NORTE & NORDESTE & $\begin{array}{l}\text { CENTRO } \\
\text { OESTE }\end{array}$ & SUDESTE & SUL \\
\hline \multicolumn{7}{|l|}{ Resolutividade } \\
\hline Boa & $5827(32,2 \%)$ & $401(31,7 \%)$ & $2723(35,4 \%)$ & $534(34,0 \%)$ & $1507(30,0 \%)$ & $662(25,9 \%)$ \\
\hline Regular & $8968(49,5 \%)$ & $443(35,1 \%)$ & $2987(38,8 \%)$ & $730(46,4 \%)$ & $3125(62,2 \%)$ & $1683(65,9 \%)$ \\
\hline Ruim & $3319(18,3 \%)$ & $419(33,2 \%)$ & $1990(25,8 \%)$ & $308(19,6 \%)$ & $395(7,9 \%)$ & $207(8,1 \%)$ \\
\hline TOTAL & $18114(100 \%)$ & $1263(100 \%)$ & $7700(100 \%)$ & $1572(100 \%)$ & $5027(100 \%)$ & $2552(100 \%)$ \\
\hline \multicolumn{7}{|l|}{$\begin{array}{l}\text { Processo de } \\
\text { Trabalho }\end{array}$} \\
\hline Boa & $8521(47,0 \%)$ & $426(33,7 \%)$ & $3964(51,5 \%)$ & $567(36,1 \%)$ & $2468(49,1 \%)$ & $1096(42,9 \%)$ \\
\hline Regular & $5355(29,6 \%)$ & $355(28,1 \%)$ & $2110(27,4 \%)$ & $492(31,3 \%)$ & $1556(31,0 \%)$ & $842(33,0 \%)$ \\
\hline Ruim & $4238(23,4 \%)$ & $482(38,2 \%)$ & $1626(21,1 \%)$ & $513(32,6 \%)$ & $1003(20,0 \%)$ & $614(24,1 \%)$ \\
\hline TOTAL & $18114(100 \%)$ & $1263(100 \%)$ & $7700(100 \%)$ & $1572(100 \%)$ & $5027(100 \%)$ & $2552(100 \%)$ \\
\hline \multicolumn{7}{|l|}{$\begin{array}{l}\text { Coordenaçáo } \\
\text { do Cuidado }\end{array}$} \\
\hline Boa & $5027(27,8 \%)$ & $588(46,6 \%)$ & $2437(31,6 \%)$ & $564(35,9 \%)$ & $986(19,6 \%)$ & $452(17,7 \%)$ \\
\hline Regular & $6414(35,4 \%)$ & $220(17,4 \%)$ & $2340(30,4 \%)$ & $390(24,8 \%)$ & $2389(47,5 \%)$ & $1075(42,1 \%)$ \\
\hline Ruim & $6673(36,8 \%)$ & $455(36,0 \%)$ & $2923(38,0 \%)$ & $618(39,3 \%)$ & $1652(32,9 \%)$ & $1025(40,2 \%)$ \\
\hline TOTAL & $18114(100 \%)$ & $1263(100 \%)$ & $7700(100 \%)$ & $1572(100 \%)$ & $5027(100 \%)$ & $2552(100 \%)$ \\
\hline \multicolumn{7}{|l|}{ Infraestrutura } \\
\hline Boa & $14805(83,6 \%)$ & $929(74,7 \%)$ & $6086(80,5 \%)$ & $1336(86,6 \%)$ & $4369(89,3 \%)$ & $2085(84,5 \%)$ \\
\hline Regular & $2415(13,6 \%)$ & $235(18,9 \%)$ & $1166(15,4 \%)$ & $189(12,2 \%)$ & $475(9,7 \%)$ & $350(14,2 \%)$ \\
\hline Ruim & $487(2,8 \%)$ & $79(6,4 \%)$ & $310(4,1 \%)$ & $18(1,2 \%)$ & $47(1,0 \%)$ & $33(1,3 \%)$ \\
\hline TOTAL & $17.707(100 \%)^{* *}$ & $1243(100 \%)^{* *}$ & $7562(100 \%)^{* *}$ & $1543(100 \%)^{* *}$ & $4891(100 \%)^{* *}$ & $2468(100 \%)^{* *}$ \\
\hline
\end{tabular}

${ }^{*}$ A análise da Saúde Bucal apresentou 407 dados ausentes, destes: 20 foram no território Norte; 138 na região Nordeste, 29 no Centro Oeste, 136 no Sudeste e por fim, 84 no território Sul.

** A dimensão infraestrutura também apresentou 407 dados ausentes, destes: 20 foram na região Norte; 138 no Nordeste, 29 no território Centro Oeste, 136 no Sudeste e por fim, 84 na região Sul.

Fonte: Próprio autor.

Dentre os resultados apresentados na Tabela 2, destacam-se os relativos à qualidade da saúde bucal. No Brasil, das equipes analisadas, 25,1\% foram consideradas boas; $53,2 \%$ regulares e $21,7 \%$ ruins. Logo, quando se analisam os territórios com as 
equipes melhor avaliadas no critério qualidade de saúde bucal, é possível observar que as equipes inseridas nas regióes Sul e Sudeste apresentaram os melhores resultados no critério qualidade da saúde bucal.

Ao tratar sobre a dimensão resolutividade, pode-se observar que o Sudeste e o Sul apresentam padróes de qualidade melhores, apresentando aproximadamente $8 \%$ de equipes classificadas como "ruim". Entretanto, o território Norte é o que apresenta o padrão de qualidade pior. As equipes consideradas boas estão inseridas no Nordeste e Centro Oeste e o maior percentual de equipes consideradas regulares, nos territórios Sudeste e Sul.

Quanto à dimensão processo de trabalho, pôde-se observar que o Sudeste e o Nordeste apresentam padróes de qualidade melhores, apresentando percentuais maiores de equipes registradas como "boa" e menores, de equipes classificadas como "ruim". Por outro lado, no território Sul estão inseridas as equipes enquadradas como "regular".

No tocante ao estudo da dimensão coordenação do cuidado, do total de equipes analisadas no país, foi possível constatar que as regiōes Norte e Centro Oeste apresentam padróes de qualidade melhores. Por outro lado, o território Sul é o que apresenta o padrão de qualidade pior, com $40,2 \%$ das equipes avaliadas com o padrão de qualidade ruim. O maior percentual de equipes consideradas "regular" estão localizadas nas regióes Sul e Sudeste.

Para o estudo da dimensão infraestrutura, ao consideramos o contexto nacional, pôde-se observar que o Sudeste, o Centro Oeste, e o Sul apresentam padrôes de qualidade melhores. Sendo o Sudeste a região que apresenta o melhor padrão de qualidade relacionado à infraestrutura. Já as regiôes Nordeste e Norte apresentam os piores padróes de qualidade, entretanto, o território Norte é o que apresenta o padrão de qualidade pior.

No que concerne à análise bivariada multinível relacionada à qualidade da saúde bucal com a variável independente (IDHM), a hipótese de que quanto melhor o IDH, melhor seria a qualidade de assistência à saúde bucal na atenção primária segundo os dados do PMAQ-AB não se confirmou. Não houve diferença significativa da qualidade da assistência à saúde bucal entre municípios com melhor IDH em relação aos de baixo IDH, conforme as informaçôes expressas na Tabela 3. 
Tabela 3. Análise bivariada entre a qualidade da saúde bucal e a variável contextual. Natal, RN, 2019.

\begin{tabular}{cccccc}
\hline Variável & Qualidade da assistência à saúde bucal \\
\hline Boa & Regular/Ruim & RP & LI (IC95\%) & LS (IC95\%) \\
\hline $\mathrm{N}(\%)$ & $\mathrm{N}(\%)$ \\
\hline \multicolumn{5}{c}{ Nivel (municípios) } \\
\hline Índice de Desenvolvimento Humano Municipal (IDH-M) \\
\hline Alto IDHM-M & $2.237(25.36 \%)$ & $6.584(74,64 \%)$ & 1 & \\
\hline Baixo IDH-M & $2.211(24,88 \%)$ & $6.675(75,12 \%)$ & 1,017 & 0,982 & 1,054 \\
\hline
\end{tabular}

Fonte: Próprio autor.

\section{Discussão}

O resultado que chama mais atenção no presente estudo é que apenas um quarto das equipes de saúde bucal são consideradas boas quanto à qualidade da assistência à saúde bucal no Brasil. Diante desta realidade, destacamos que esta é uma situação preocupante, principalmente, ao se considerar que os critérios utilizados se referem a condiçôes mínimas na assistência. É possível observar que a magnitude das diferenças regionais ainda se manifesta nas dimensôes analisadas, pois a regiáo sudeste apresentou supremacia em relação às demais em todas a as dimensões analisadas. No critério "resolutividade", vem seguida da região Sul; no critério "processo de trabalho", pela regiâo Nordeste; na "coordenação do cuidado", pelo território Norte e por fim na "Infraestrutura" pelo Centro Oeste. No entanto, quando analisado o critério "qualidade de saúde bucal no Brasil", a regiấo Sul obteve o melhor percentual dentre as regióes citadas, quando são analisados os critérios bom e regular agregados.

Neste sentido, em um estudo análogo publicado por Miclos et al. (2015) sobre avaliação do desempenho da atenção básica nos municípios brasileiros observa-se que o Sul apresenta maior percentual dos resultados satisfatórios, seguido da regiáo Sudeste, enquanto a região Norte apresenta-se com o menor percentual de municípios nessa condição (MICLOS et al., 2015). Estes achados corroboram com os resultados encontrados na nossa pesquisa, pois a qualidade da assistência é pior na regiāo Norte e melhor no Sul e Sudeste. Destaca-se que cuidados de alta qualidade envolvem uma avaliação completa, detecção de condiçôes assintomáticas e coexistentes, diagnóstico 
preciso, tratamento adequado e oportuno, encaminhamento quando necessário para cuidados hospitalares e cirurgia, e a capacidade de acompanhar o paciente e ajustar o tratamento, conforme necessário (KRUK et al., 2018).

Como a dimensão acesso não gerou variável latente, abordaremos alguns aspectos importantes para termos a excluído deste estudo. Neste ponto cabem algumas consideraçôes sobre acesso que podem provocar uma discussão mais ampliada sobre esta dimensão. Neste estudo, a dimensão "acesso" não foi usada para criar o indicador de qualidade geral, pois não gerou variável latente. No campo da saúde bucal, há discussão da influência do acesso na qualidade da assistência, uma vez que o acesso aos serviços odontológicos não necessariamente se traduz em melhoria da saúde bucal dos indivíduos. Em recente estudo, Ely et al. (2016) sugerem que a expansão do acesso com a inserção de equipes de saúde bucal não se traduziu em melhor saúde bucal, pois observaram que jovens de áreas não cobertas por equipes de saúde bucal perderam menos dentes que adolescentes inseridos em áreas cobertas por equipes de saúde bucal (ELY et al., 2016).

Além disso, Chaves et al. (2017) relatam que o esforço para ampliação do financiamento, infraestrutura e recursos humanos náo produziu ampliaçáo em termos de acesso, pois não houve aumento da cobertura da primeira consulta odontológica (indicador que reflete acesso ao sistema público) e esta é uma questão problemática, portanto, merece melhor aprofundamento (CHAVES et al., 2017).

É importante reforçar que acesso sem qualidade pode ser considerado uma promessa de cobertura universal de saúde vazia (OMS, 2018). Além do mais, historicamente, o acesso dos brasileiros à saúde bucal era extremamente difícil e limitado, fazendo com que as pessoas se acostumassem a só procurar atendimento odontológico em casos de dor (BRASIL, 2016). Assim sendo, é necessário o esforço coletivo da gestão e das Equipes da Saúde Bucal para a construção efetiva e de qualidade da dimensão acesso, através da reorganização do processo de trabalho de acordo as necessidades do território e criação de formas participativas que denotem a ampliação da porta de entrada. Fahel, Silva e Xavier (2018) relatam que o desafio persiste e reside na construção de uma rede de cuidados à saúde bucal coerente com os princípios do SUS, que possa garantir os cuidados nos três níveis de atenção (FAHEL, SILVA, XAVIER, 2018).

Na dimensão processo de trabalho, o Nordeste apresentou o melhor padrão de qualidade. Este desenho traz a leitura que o padrão de qualidade já está melhorando 
em algumas áreas. Uma das explicaçôes para isso pode estar relacionada à implantação da ESF que se iniciou no estado Ceará (localizado no Nordeste) através da experiência do Programa de Agentes Comunitários de Saúde e expandida com a incorporação de médicos e enfermeiros (MENDES, 2015). O objetivo de qualificar o trabalho e não apenas melhorar dados estatísticos pode ter sido um dos pontos fundamentais para trazer o Nordeste com uma boa qualidade na dimensão processo de trabalho.

Cabe ressaltar também que os padrôes de processo de trabalho que formaram a variável latente para a análise da qualidade são de fundamental importância para que o processo de trabalho se concretize. Entre eles: monitoramento e análise dos indicadores; planejamento e programação das atividades e apoio da gestão. É importante salientar que em estudo análogo, realizado em Pernambuco e publicado recentemente por Viana et al.(2019) sobre avaliação da qualidade da assistência em saúde bucal na AP, também com dados secundários do segundo ciclo do PMAQ$\mathrm{AB}$, os resultados corroboram com os achados desta pesquisa ao serem identificadas correlaçôes entre os indicadores de urgência odontológica, cobertura de escovação dental supervisionada e tratamentos concluídos nos estratos de qualidade relacionados ao processo de trabalho das equipes de Saúde Bucal (VIANA et al., 2019).

Ceccim (2005) afirma que se torna crucial o desenvolvimento de recursos tecnológicos de operação do trabalho ordenados pela noção de aprender a aprender, de trabalhar em equipe, de construir cotidianos eles mesmos como objeto de aprendizagem individual, coletiva e institucional. E ainda sugere a construção de equipes multiprofissionais, coletivos de trabalho, lógicas apoiadoras uns nos outros na equipe e orientadas para a resolutividade dos problemas de saúde das populaçôes, caso contrário será colocada em risco a qualidade do trabalho (CECCIM, 2005). Logo, é necessário pontuar que a disponibilidade de pessoal não garante por si só um bom atendimento. A prioridade é o contínuo desenvolvimento profissional para garantir que os profissionais de saúde mantenham e melhorem seus conhecimentos e habilidades - abrangendo uma ampla gama de competências no trabalho (OMS, 2018).

No tocante à resolutividade, um estudo sobre a comparação das práticas odontológicas segundo modelos de atendimento tradicional e Saúde da Família em Pelotas no Rio Grande do Sul relata que há diferenças favoráveis ao grupo Equipes de Saúde Bucal (eSB), na média da razão de tratamentos concluídos/ primeiras consultas odontológicas programáticas. Os autores também afirmam que 
a resolução da necessidade deve incluir o tratamento concluído, ou seja, a relação entre tratamentos concluídos e primeiras consultas odontológicas programáticas (THUROW, CASTILHOS e COSTA, 2015). Neste mesmo contexto, em uma pesquisa realizada em Marília (SP), Bulgareli et al. (2014) afirmam que fica evidente que as unidades de saúde da família apresentaram melhores índices de resolutividade que as unidades tradicionais.

Ainda neste contexto, Rodrigues et al. (2012) identificaram inquietaçóes de grande parte dos profissionais em tornar a assistência resolutiva e preocupação com o retorno dos usuários ao serviço, para dar continuidade ao tratamento; prioridades de atendimento da demanda espontânea, com ênfase nas emergências e urgências e dificuldade em realizar levantamentos para reconhecer as necessidades da população adscrita a sua unidade de saúde.

Cabe destacar que em uma análise descritiva do PMAQ-AB em Pernambuco observou-se que a maioria das equipes de saúde bucal se refere à disponibilidade dos equipamentos necessários para a realização das ações e serviços de saúde bucal. Em pouco menos de $20 \%$ das equipes, a disponibilidade de insumos odontológicos para procedimentos restauradores foi limitada (LORENA SOBRINHO et al., 2015). Estes achados corroboram com os resultados encontrados na nossa pesquisa, pois, a infraestrutura é considerada "boa" (80,5\%) nos estados do Nordeste.

Ainda neste contexto, é importante relatar que, embora insumos como medicamentos e equipamentos sejam comumente contados em pesquisas, estes são fracamente relacionados à qualidade de atendimento que as pessoas recebem (KRUK et al., 2018). Ou seja, infraestrutura adequada não equivale necessariamente a cuidados de alta qualidade (OMS, 2018). No mais, Corrêa e Celeste (2015) ressaltam que um mínimo de estrutura é necessário, mas não suficiente para garantir cuidados de elevada qualidade, pois estes também dependem de outros fatores. Os resultados encontrados nesta pesquisa corroboram com os autores supracitados, pois houve poucas disparidades regionais ao analizarmos esta dimensão.

Cabe ressaltar que os padróes de infraestrutura que formaram a variável latente para a análise da qualidade são os equipamentos e insumos fundamentais para o pleno funcionamento do consultório odontológico. É válido destacar também que nos achados de Neves, Giordani e Hugo (2019), em relação às variáveis elencadas no nível da equipe de saúde e dos equipamentos e insumos para atenção odontológica, observou-se maior prevalência de realização de procedimentos odontológicos 
curativos quando havia alta e baixa rotação (NEVES, GIORDANI E HUGO, 2019). Lorena Sobrinho et al. (2015), observaram que a maioria dos consultórios odontológicos apresentaram disponibilidade dos equipamentos necessários para a realização das açôes e serviços de saúde bucal, com exceção da autoclave exclusiva para odontologia, o qual não fora identificada em $65 \%$ das unidades (LORENA SOBRINHO et al., 2015).

Para a coordenação do cuidado, o maior percentual de equipes consideradas boas está inserido na regiáo Norte. Por outro lado, para esta mesma dimensão observamos que os maiores percentuais de equipes consideradas ruins estão inseridos no território sul. Concernente ao contexto citado, Casotti et al. (2014) sugerem que o alto percentual de respostas afirmativas quanto à garantia da continuidade do tratamento precisa ser mais bem estudado, pois não condiz com os resultados encontrados sobre perfil do agendamento na atenção primária e sobre o acesso a outros níveis de complexidade, particularmente nas regiôes Norte e Nordeste (CASOTTI et al., 2014). Da mesma forma que os autores supracitados, é de fundamental importância a realização de novas pesquisas com outras abordagens metodológicas para verificar estes achados.

Para Mendes (2015), as falhas na coordenação do cuidado ocorrem, em geral, na transição de uma unidade de saúde a outra, no caso entre a ESF e a unidade de AAE (Atenção Ambulatorial Especializada), e manifestam-se por problemas de responsabilização e de quebra do fluxo de informaçóes (MENDES, 2015). Em seus achados, Starfield (2002) relata que na maioria dos estudos, a coordenação da atenção é considerada fraca, é por isso também que a autora relata que alcançar a coordenação é um desafio para profissionais de atenção primária devido a fatores como: multiplicidade de tipos de motivos para encaminhamento e das dificuldades técnicas na transferência e reconhecimento de informaçôes geradas em diferentes lugares (STARFIELD, 2002).

A hipótese de que quanto melhor o IDHM, melhor seria a qualidade de assistência à saúde bucal na atenção primária, segundo os dados do PMAQ-AB, não se confirmou. A diferença pode não ter sido encontrada porque ao analisarmos o padrão regular na mesma categoria do ruim pode ter mascarado a diferença de baixo IDH com alta cobertura da saúde da família. $\mathrm{Na}$ análise bivariada multinível da variável independente (IDHM) com a variável dependente (qualidade de saúde bucal), os resultados encontrados revelam que estatisticamente não há diferença significativa entre os municípios com alto e baixo IDHM. 
Recentemente, Tomasi et al. (2017) encontraram resultados que corroboram com os nossos achados e afirmam que seria esperado que um padrão contrário ao do IDH fosse observado, e que quanto maior o número de ESF, mais qualidade na atenção às gestantes, mas as diferenças não apresentaram nenhum padrão nem foram significativas estatisticamente (TOMASI et al., 2017).

Embora não tenhamos encontrado achados significativos ao associar o IDH à qualidade de assistência à saúde bucal, um ponto que precisa ser discutido é a desigualdade social, pois está fortemente associada às dimensóes que estudamos para construir a variável qualidade de saúde bucal. Peres et al. (2012) relatam que as desigualdades entre os grupos sociais ainda são grandes e podem ser consideradas ética e politicamente inaceitáveis, uma vez que revelam desvantagens de acesso e de utilização dos serviços odontológicos entre os mais pobres (PERES et al., 2012).

Assim sendo, é possível compreender as relaçōes de desigualdade, em seu sentido mais amplo, como construçôes sociais localizadas historicamente e estabelecidas de forma arbitrária em diferentes momentos históricos e sociais. Entre o rol de justificativas para essas diferenciaçôes está a localização geográfica (rural/urbano, norte/sul, centro/periferia) (MELLO, 2018). Logo, as proporçôes de pessoas que nunca foram ao dentista segundo renda são mais desiguais entre os mais jovens, o que agrava a situação, considerando que os principais agravos bucais, como a cárie dentária, iniciam-se precocemente, atingindo a dentição decídua (PERES et al., 2012).

É oportuno ressaltar também que a qualidade deficiente dos cuidados não contribui apenas para o ônus global das doenças e necessidades de saúde não atendidas, mas também exerce um impacto econômico substancial, com consideráveis implicaçóes de custo para sistemas de saúde e comunidades em todo o mundo (OMS, 2018). Embora os sistemas de saúde tenham uma aparência diferente e em distintas configuraçóes, todas as pessoas devem poder contar com o recebimento cuidados de alta qualidade que melhorarão sua saúde e ganharão sua confiança. É hora de repensar nossas abordagens passadas: cobrar e investir mais neste crucial determinante da saúde (KRUK et al., 2018).

As limitaçôes deste estudo estão relacionadas ao uso de dados secundários e que eles se referem somente às equipes com saúde bucal que aderiram ao PMAQ-AB no segundo ciclo e não ao total de unidades básicas de saúde no Brasil. 


\section{Conclusões}

O principal achado deste estudo é que apenas $25 \%$ das equipes de saúde bucal que aderiram ao PMAQ-AB são consideradas boas referente à qualidade da assistência à saúde bucal no Brasil. Outra descoberta importante é que não há diferença estatisticamente significativa na análise da qualidade entre os municípios com alto e baixo IDH. Analisar a qualidade da saúde bucal deve ser objeto de trabalho dos profissionais de saúde, gestores, formuladores de política, governo e sociedade civil para que, de fato, se realize a melhora contínua da qualidade dos cuidados em saúde.

No mais, é imprescindível estimular a revisão dos processos de trabalho e planejamento exercidos pelas eSB, bem como a realizar, de fato, a coordenação do cuidado, para que as açôes efetivamente transformem as condiçóes de saúde bucal e melhorem a qualidade de vida da população.

\section{Referências}

AQUILANTE, A. G.; ACIOLE, G. G. O cuidado em saúde bucal após a Política Nacional de Saúde Bucal - "Brasil Sorridente": um estudo de caso. Ciênc. Saúde Coletiva, Rio de Janeiro, v. 20, n. 1, p.239-248, jan. 2015.

BRASIL. Ministério da Saúde. Passo a Passo das Açóes da Politica Nacional de Saúde Bucal. Brasília: Ministério da Saúde, 2016.

BRASIL. Ministério da Saúde. Pesquisa Nacional de Saúde Bucal SB Brasil 2010. Brasília: Ministério da Saúde, 2011.

BRASIL. Ministério da Saúde. Relatórios Analíticos. Disponível em: < http://pmaq.lais.huol. ufrn.br/relat_analiticos/inicial/relatorios//\#> Acesso em: 06 agosto 2019.

BRASIL. Programa Nacional de Melhoria do Acesso e da Qualidade da Atençáo Básica (PMAQ). Manual Instrutivo $3^{\circ}$ Ciclo (2015 - 2016). Brasília: Ministério da Saúde, 2015.

BULGARELI, J. et al. A resolutividade em saúde bucal na atenção básica como instrumento para avaliação dos modelos de atenção. Ciência \& Saúde Coletiva, v.19, n.2, p.383-391, 2014.

CASOTTI, E.; CONTARATO, P. C.; FONSECA, A. B. M.; BORGES, P. K. O.; BALDANI, M. H. Atenção em Saúde Bucal no Brasil: uma análise a partir da Avaliação Externa do PMAQAB. Saúde debate, v. 38, n. especial, p. 140-157, out. 2014.

CECCIM, R.B. Educaçáo Permanente em Saúde: desafio ambicioso e necessário. Interface Comunic, Saúde, Educ, v.9, n.16, p.161-77, set.2004/fev.2005. 
CHAVES,S.C.et al. Política de Saúde Bucal no Brasil 2003-2014: cenário, propostas, açôes e resultados. Ciência \& Saúde Coletiva. v.22, n.6, p.1791-1803, 2017.

CORRÊA, G. T.; CELESTE, R. K. Associação entre a cobertura de equipes de saúde bucal na saúde da família e o aumento na produção ambulatorial dos municípios brasileiros, 1999 e 2011. Cad. Saúde Pública, v. 31, n.12, p.2588-2598, dez. 2015.

ELY,H.C; ABEGG. C; CELESTE, R.K; PATTUSSI, M.P. Impacto das equipes de saúde bucal da Estratégia da Saúde da Família na saúde bucal de adolescentes do sul do Brasil. Ciência \& Saúde Coletiva, v.21, n.5, p.1607-1616, 2016.

FAHEL, M.; SILVA, M. P.; XAVIER, D. O. A trajetória da atenção primária à saúde no Brasil: De Alma Ata ao Programa Mais Médicos. Belo Horizonte: Fundação João Pinheiro, 2018.

IPEA, Instituto de Pesquisa Econômica Aplicada. Radar IDHM : evolução do IDHM e de seus indices componentes no período de 2012 a 2017. - Brasília : IPEA : PNUD : FJP, 2019.

KRUK, M. E; GAGE, A. D; ARSENAULT, C. et al. High-quality health systems in the Sustainable Development Goals era: time for a revolution. The Lancet Global Health Commission, v. 18, n. 30386-3, p. 1-57, 2018. Dói: http://dx.doi.org/10.1016/S2214-109X.

LORENA SOBRINHO, J. E.; MARTELLI, P. J. L.; ALBUQUERQUE, M. S. V.; LYRA, T. M.; FARIAS, S. F. Acesso e qualidade: avaliação das equipes de saúde bucal participantes do PMAQ-AB 2012 em Pernambuco. Saúde Debate|Rio de Janeiro, v. 39, n. 104, p. 136-146, 2015.

MELLO, J. Estratégias de superação da pobreza no Brasil e impactos no meio rural. Rio de janeiro: Ipea, 2018.

MENDES, E. V. A Construçâo Social da Atenção Primária à Saúde. Brasília: CONASS, 2015.

MICLOS, P.V.; CALVO, M.C.M.; COLUSSI, C.F.; Avaliação do desempenho da Atenção Básica nos municípios brasileiros com indicador sintético. Saúde debate. v.39, n.107, p.984-996, 2015.

NEVES, M.; GIORDANI, J. M. A; HUGO, F.N. Atenção primária à saúde bucal no Brasil: processo de trabalho das equipes de saúde bucal. Ciência \& Saúde Coletiva, v.24, n.5, p.1809$1820,2019$.

OMS. Delivering quality health services: a global imperative for universal health coverage. Geneva: World Health Organization, Organisation for Economic Co-operation and Development, and The World Bank. Licence: CC BY-NC-SA 3.0 IGO, 2018.

PERES, K.G. et al. Redução das desigualdades sociais na utilização de serviços odontológicos no Brasil entre 1998 e 2008.Rev Saúde Pública.v.46, n.2, p.250-258, 2012.

Programa das Nações Unidas para o Desenvolvimento (PNUD). Atlas do desenvolvimento humano dos municípios. PNUD; 2013. Disponível em: <http://www.atlasbrasil.org.br/2013/pt/ ranking\#> Acesso em: 04 de julho 2019. 
RODRIGUES, A. A. A. Oliveira et al. SAÚDE BUCAL NA ESTRATÉGIA SAÚDE DA FAMÍLIA EM UM MUNICÍPIO DO SEMIÁRIDO BAIANO. Revista Baiana de Saúde Pública, [S.l.], v. 35, n. 3, p. 695 - 709, set. 2012. ISSN 2318-2660. Disponível em: <http:// rbsp.sesab.ba.gov.br/index.php/rbsp/article/view/326>. Acesso em: 26 ago. 2019. doi: https:// doi.org/10.22278/2318-2660.2011.v35.n3.a326.

SCHERER, C. I.; SCHERER, M.D.A. Avanços e desafios da saúde bucal após uma década de Programa Brasil Sorridente. Rev Saúde Pública, v.49, n. 98, p.1-12, 2015.

STARFIELD, B. Atenção Primária: equilíbrio entre necessidades de saúde, serviços e tecnologia. Brasília: UNESCO, Ministério da Saúde, 2002. 726 p.

THUROW, L. L; CASTILHOS, E. D.; COSTA, J. S. D. Comparação das práticas odontológicas segundo modelos de atendimento: tradicional e da Saúde da Família, Pelotas-RS, 2012-2013. Epidemiol. Serv. Saúde, v. 24, n. 3, p. 545-550, 2015.

TOMASI, Elaine. et al. Qualidade da atenção pré-natal na rede básica de saúde do Brasil: indicadores e desigualdades sociais. Cad. Saúde Pública, v.33, n.3, p.1-11, 2017

VIANA, I.B.; MOREIRA RS, MARTELLI, P.J.L.; OLIVEIRA, A.L.S.; MONTEIRO, I.S. Avaliação da qualidade da assistência em saúde bucal na Atenção Primária à Saúde em Pernambuco, 2014*. Epidemiol. Serv. Saude, v.28, n.2, p.1-12, 2019. doi: 10.5123/S167949742019000200015 


\section{Abstract}

\section{Oral Care Quality in Brazilian Primary Care}

The aim of this study was to assess the quality of oral care provided in Brazilian primary care and identify associated contextual socioeconomic factors. This is a cross-sectional study whose unit of analysis was all the oral care teams that participated in an external assessment for the second cycle of the Access and Quality Improvement Program - Primary Care. Quality was measured in the following dimensions: access, resolution, work process, care coordination and infrastructure. The quality criteria of the external assessment were grouped into these dimensions and weighted using Item Response Theory. The indicator of oral care quality was the result of grouping latent variables. Descriptive analyses were performed by region and association between "Oral Care Quality" and the Municipal Human Development Index (HDI), with $5 \%$ significance. Only $25 \%$ of the oral care teams were considered good. The infrastructure dimension obtained the best results and care coordination the worst. There was no statistically significant difference between municipalities with high and low HDIs. Quality assessment is vital to ensure the continuous improvement of quality healthcare.

Keywords: Oral Health; Care Quality Assessment; Primary Care. 